\title{
Genetic Stability of Magnaporthe oryzae during Successive Passages through Rice Plants and on Artificial Medium
}

\author{
Sook-Young Park ${ }^{1}$, Myoung-Hwan Chi', Michael G. Milgroom ${ }^{2}$, Hyojung Kim ${ }^{1}$, Seong-Sook Han ${ }^{3}$, \\ Seogchan Kang ${ }^{4}$ and Yong-Hwan Lee ${ }^{1 *}$ \\ ${ }^{\prime}$ Department of Agricultural Biotechnology, Center for Fungal Genetic Resources, and Center for Fungal Pathogenesis, Seoul \\ National University, Seoul 151-921, Korea \\ ${ }^{2}$ Department of Plant Pathology and Plant-Microbe Biology, Cornell University, Ithaca, NY 14853, USA \\ ${ }^{3}$ Agricultural Microbiology Team, National Academy of Agricultural Science, RDA, Suwon 441-707, Korea \\ ${ }^{4}$ Department of Plant Pathology, The Pennsylvania State University, University Park 16802, USA
}

(Received on September 27, 2010; Accepted on November 2, 2010)

Genetic instability of the rice blast fungus Magnaporthe oryzae has been suggested as a major factor underlying the rapid breakdown of host resistance in the field. However, little information is available on the mechanism of genetic instability. In this study, we assessed the stability of repetitive DNA elements and several key phenotypic traits important for pathogenesis after serially transferring two isolates though rice plants and an artificial medium. Using isolate $70-15$, we obtained a total of 176 single-spore isolates from 10 successive rounds of culturing on artificial medium. Another 20 isolates were obtained from germ tubes formed at the basal and apical cells of 10 three-celled conidia. Additionally, 60 isolates were obtained from isolate $\mathrm{KJ201}$ after serial transfers through rice plants and an artificial medium. No apparent differences in phenotypes, including mycelial growth, conidial morphologies, conidiation, conidial germination, appressorium formation, and virulence, or in DNA fingerprints using MGR586, MAGGY, Pot 2, LINE, MG-SINE and PWL2 as probes were observed among isolates from the same parent isolate. Southern hybridization and sequence analysis of two avirulence genes, $A V R$-Pita 1 and $A V R$-Pikm, showed that both genes were also maintained stably during 10 successive generations on medium and plants. However, one reversible loss of restriction fragments was found in the telomere-linked helicase gene (TLH1) family, suggesting some telomere regions may be more unstable than the rest of the genome. Taken together, our results suggest that phenotype and genotype of $M$. oryzae isolates do not noticeably change, at least up to 10 successive generations on a cultural medium and in host plants.

Keywords : genetic stability, genotypes, Magnaporthe grisea, phenotypes, repeat sequences

\footnotetext{
*Corresponding author.

Phone) +82-2-880-4674, FAX) +82-2-873-2317

E-mail) yonglee@snu.ac.kr
}

Magnaporthe oryzae is a heterothallic ascomycete fungus that causes rice blast, one of the most devastating diseases in rice throughout the world. Since there is no evidence for sexual reproduction in the field in most parts of the world, asexual spores (conidia) are the most important source of inoculum. Through the production of conidia, M. oryzae normally undergoes 8-11 disease cycles in rice plants under field conditions during one growing season (Han et al., 1997). Although asexual reproduction is expected to produce genetically identical clones, the high rate of appearance of new races with the ability to infect previously resistant varieties of rice, suggests that $M$. oryzae may have high mutation rates in avirulence genes, which determine race (also called pathotype in M. oryzae) (Kang et al., 2001; Kang and Lee, 2000; Zhou et al., 2007).

Although the most effective control strategy for rice blast is cultivating resistant varieties, it has often achieved only short-term success due to frequent breakdown of newly introduced resistance. This resistance breakdown has been attributed to genetic variability in M. oryzae (Bonman et al., 1986; Correa-Victoria and Zeigler, 1993; Correa-Victoria and Zeigler, 1993). Various mechanisms have been suggested to explain the frequent generation of race variants, including heterokaryosis, parasexuality, and mutations (Genovesi and Magill, 1976; Wu and Magill, 1995; Xia and Correll, 1995; Zeigler et al., 1997). Parasexual recombination of genetic markers, resulting new genetic variants, has been demonstrated in the laboratory for M. oryzae, suggesting the potential for parasexual recombination to generate new races (Noguchi et al., 2006; Zeigler et al., 1997). However, no evidence for parasexuality has been found in nature for M. oryzae. The activity of transposable DNA elements is another possible mechanism that has been suggested (Kang et al., 2001; Nishimura et al., 2000; Zhou et al., 2007). For example, mobilization of Pot2, Pot3, and LINE elements has been shown to cause variation by inserting into promoter region or coding sequences of avirulence 
genes (Kachroo et al., 1994; Kachroo et al., 1995; Kang et al., 2001; Nishimura et al., 2000), indicating their potential to generate novel races.

The transposable elements MGR586 and MAGGY have been frequently used as markers to assess genetic diversity and variation in populations of M. oryzae (Xia and Correll, 1995; Zeigler et al., 1997), mainly because 40-50 copies these elements are scattered throughout the genome in rice pathogenic isolates and appear to mutate at relatively high rates compared to other markers (Borromeo et al., 1993; Chen et al., 1995; Levy et al., 1993; Park et al., 2003; Xia et al., 1993; Xia et al., 2000). Other repetitive DNA elements in M. oryzae, such as Pot2, Pot3, LINE, and MG-SINE, are also distributed throughout the genome and have potential to be used as markers for detecting genetic variation in $M$. oryzae.

Our overall question was how stable $M$. oryzae isolates would be both genotypically and phenotypically during 10 serial transfers, which would be roughly equivalent to 10 disease cycles during an epidemic in the field in one growing season. To address this question, we serially transferred isolate 70-15, whose genome was recently sequenced (Dean et al., 2005), and field isolate KJ201 on an artificial medium and/or through rice plants and genotyped the conidia after each transfer. The specific objectives were: (i) to determine phenotypic variation of these two isolates during serial transfers in vitro and in planta, (ii) to investigate genotypic variation of the isolates collected in each generation using DNA fingerprinting and (iii) to compare sequence variation of two avr genes of isolate KJ201 isolates during successive passages in vitro and in vivo.

\section{Materials and Methods}

Culture media. Oatmeal agar medium (50 g rolled oats and 15-20 g agar per liter $\mathrm{H}_{2} \mathrm{O}$ ) was used for long-term storage and production of conidia. For long-term storage, all isolates were grown and kept as described previously (Park et al., 2003). All isolates were cultured in complete liquid medium (6 g yeast extract, $6 \mathrm{~g}$ casamino acid, $10 \mathrm{~g}$ sucrose per liter $\mathrm{H}_{2} \mathrm{O}$ ) to harvest mycelia for genomic DNA extraction as described previously (Park et al., 2003). Complete medium (CM) and oatmeal agar medium (OMA) were used to measure the mycelial growth rate and colony characteristics of individual isolates, respectively, as described below. V8 juice agar medium ( $80 \mathrm{ml}$ V8 juice, 920 $\mathrm{ml}$ distilled water, $15 \mathrm{~g}$ agar, $\mathrm{pH}$ 6.97) was used for quantifying production of conidia.

Serial transfers on artificial medium and through rice plants. Magnaporthe oryzae isolates 70-15 and KJ201 were obtained from the Center for Fungal Genetic Resources
(CFGR) at Seoul National University, Seoul, Korea.

For serial transfer on artificial medium, we used both isolates. A schematic diagram of the serial transfers of single conidia is shown in Fig. 1A. For the first generation, conidia of isolates 70-15 and KJ201 from cultures on OMA were spread on $2 \%$ water agar and incubated for $12 \mathrm{~h}$. Three germinated conidia were transferred to OMA and were grown for 2 weeks at $23^{\circ} \mathrm{C}$ under continuous fluorescent light. For the second generation, three conidia for 70 15 and one conidium from KJ201 from each of the three cultures were transferred to new plates resulting in nine isolates and three isolates, respectively. In the third generation for isolate $70-15$, two conidia from each the nine isolates were transferred, resulting in 18 isolates. From fourth to tenth generations for isolate $70-15$, one conidium from each of the 18 cultures was transferred to new plates, maintaining 18 independent replicates. For isolate KJ201, one conidium from each the three isolates of second generation of KJ201 were transferred to new plates from the third to tenth generation (Fig. 1A), maintaining three independent replicates.

For serial transfers on host plants, we only used isolate KJ201. After culturing KJ201 on OMA, conidia were collected by flooding plates with $3 \mathrm{ml}$ sterile water with 200 ppm of Tween 20, filtering through cheesecloth to remove mycelium and adjusting the concentration of the spore suspension to $5 \times 10^{5}$ conidia $/ \mathrm{ml}$. Three susceptible rice plants of the cultivar Nakdongbyeo, which is not known to contain resistance gene effective against rice blast in Korea, were grown in $20 \times 1 \mathrm{~cm}$ test tubes (to prevent crosscontamination) using Murashige-Skoog medium (Duchefa Biochemie, Haarlem, The Netherlands) with $0.8 \%$ (w/v) agar in a growth chamber at $25^{\circ} \mathrm{C}$ with a photoperiod of 16 $\mathrm{h}$ light, $8 \mathrm{~h}$ dark. Two-week old plants were inoculated with $5 \mathrm{ml}$ of conidial suspension of isolate $\mathrm{KJ} 201$ by disposable sprayer for first passages. Three plants were inoculated for each of three independent replicates (Fig. 1B). Seven days after inoculation, one leaf per plant for a total of 3 leaves for each replicate was sampled, surface sterilized and placed on a water agar plate. Three days later, a single conidium from each of the three plates was transferred to OMA for genotypic and phenotypic assays (Fig. 1B). In parallel, approximately 2,000 to 3,000 conidia produced on the detached leaves were separately collected from each of the three plates of the leaves in $3 \mathrm{ml}$ of $200 \mathrm{ppm}$ Tween 20 solution, as described above. Each conidial suspension was used to inoculate another three host plants as an independent replicate. Ten generations of inoculation and conidial isolation were performed. To distinguish the isolates generated by serial transfers on medium from those obtained by plant inoculations, we added $\mathrm{M}$ (for medium) and $\mathrm{H}$ (for host), respectively, after the parental isolate name (Table 1). 


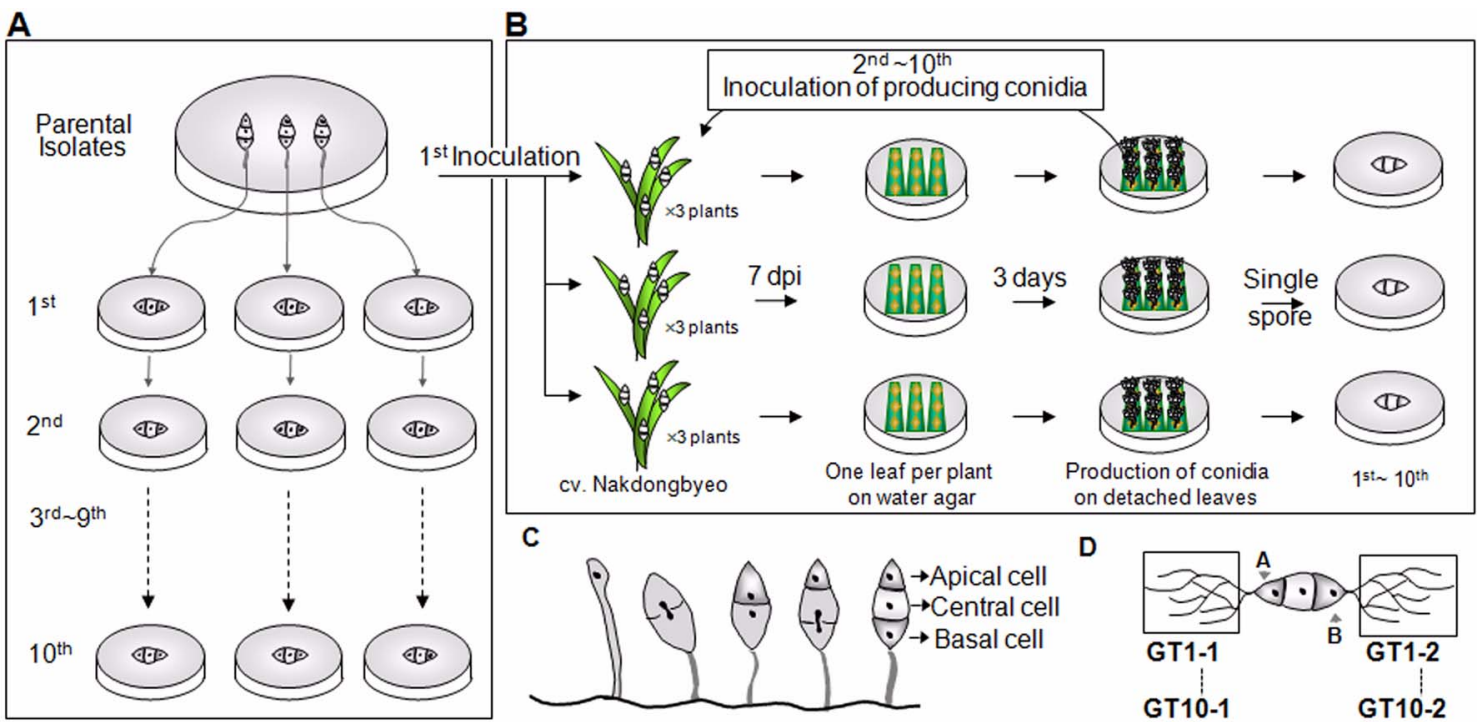

Fig. 1. Serial transfer and isolation methods used in this study. Schematic diagram of successive transfers of Magnaporthe oryzae isolate KJ201 (A) on artificial medium and (B) through rice plants. (C) Conidiogenesis of M. oryzae showing the origin of nuclei in each cell (Rao, 1994). (D) Methods for isolating from germinated conidia to produce 70-15 GT (germ tube) isolates; A and B in this panel denote the apical and basal cells of a conidium, respectively.

In addition to serial transfers, we assessed stability by sampling germ tube isolates from both ends of the same conidium. Conidia of isolate $70-15$ were spread on $2 \%$ water agar for $24 \mathrm{~h}$ at room temperature and allowed to germinate. Germ tubes at both the basal and apical cells of each of 10 conidia, which typically contain three-cells (Fig. $1 \mathrm{C})$, were carefully cut from the medium under a light microscope $(100 \times)$ and transferred to OMA (Fig. 1D). Twenty isolates, one each from the apex and base of 10 conidia, named 70-15-GT (germ tube), were recovered for characterization.

In vitro phenotypic assays for growth rate, colony morphology, conidiation, conidial morphology, conidial germination and appressorium formation in vitro. $3-\mathrm{mm}$ diameter disks from the margin of 14-d-old cultures on OMA were placed on CM and OMA to determine growth and colony morphology, respectively. The cultures were incubated at $25^{\circ} \mathrm{C}$ under constant fluorescent light with three replicates. After 5 days, the diameter of each colony was measured on CM and colony morphology was observed on OMA. Conidia were harvested from 14-d-old cultures on OMA using sterile distilled water and counted with a hemocytometer. Conidial morphology, conidial germination rate, and appressorium formation were observed on glass cover slips as described previously (Jeon et al., 2008). All experiments were repeated at least twice independently with three replicates, and a representative set of data was represented.
Virulence assay. Isolate $\mathrm{KJ} 201$ and single-conidial isolates from each of the three replicates from the $1^{\text {st }}, 5^{\text {th }}$, and $10^{\text {th }}$ passage generations through rice plants and on artificial medium were compared for virulence. Conidia were harvested from 14-d-old cultures on OMA as described above and $5 \mathrm{ml}$ of conidial suspension $\left(4 \times 10^{5}\right.$ conidia $\left./ \mathrm{ml}\right)$ was sprayed onto seedlings of cv. Nakdongbyeo at the 3 4-leaf stage grown in a growth chamber at $25^{\circ} \mathrm{C}$ with a photoperiod of $16 \mathrm{~h}$. The inoculated plants were kept in a dew chamber with $100 \%$ relative humidity for $24 \mathrm{~h}$, and then transferred to a growth chamber at $25^{\circ} \mathrm{C}$ with a photoperiod of $16 \mathrm{~h}$. Disease reactions were scored 7 days after inoculation using the 0 to 9 disease index defined by Valent et al. (1991). All experiments were performed at least twice.

DNA extraction, Southern blot hybridization and DNA sequencing. Culturing of $M$. oryzae, genomic DNA extraction and DNA fingerprinting analyses with probes MGR586, MAGGY, Pot2, Mg-SINE, Line, TLH1, PWL2 and AVRPital were done as described previously (Park et al., 2003).

The two PCR primer pairs used to amplify the coding sequences of AVR-Pital and AVR-Pikm are shown in Table 2. The PCR-cycling program included an initial denaturation for $2 \mathrm{~min}$ at $95^{\circ} \mathrm{C}, 30$ cycles of $30 \mathrm{sec}$ denaturation at $94^{\circ} \mathrm{C}, 30 \mathrm{sec}$ annealing at $56^{\circ} \mathrm{C}$, and $1 \mathrm{~min}$ extension at $72^{\circ} \mathrm{C}$, followed by a final extension for $5 \mathrm{~min}$ at $72^{\circ} \mathrm{C}$. The PCR products were purified using MEGA-spin ${ }^{\mathrm{TM}}$ PCR Product Purification Kit (Intron Biotechnology, Korea). All products were sequenced from both directions using the same PCR primers. 
Table 1. Isolates used in this study

\begin{tabular}{lccc}
\hline \hline Isolates & Generation & Substrate & $\begin{array}{c}\text { Total No. } \\
\text { of isolates }\end{array}$ \\
\hline $70-15$ & Parental isolate & 1 \\
$70-15-M^{\text {b }} 1-1 \sim 3$ & $1^{\text {st }}$ & Artificial medium & 3 \\
$70-15-\mathrm{M} 2-1 \sim 3$ & $2^{\text {nd }}$ & Artificial medium & 9 \\
$70-15-\mathrm{M} 3-1 \sim 9$ & $3^{\text {rd }}$ & Artificial medium & 18 \\
$70-15-\mathrm{M} 4-1 \sim 18$ & $4^{\text {th }}$ & Artificial medium & 18 \\
$70-15-\mathrm{M} 5-1 \sim 18$ & $5^{\text {th }}$ & Artificial medium & 18 \\
$70-15-\mathrm{M} 6-1 \sim 18$ & $6^{\text {th }}$ & Artificial medium & 18 \\
$70-15-\mathrm{M} 7-1 \sim 18$ & $7^{\text {th }}$ & Artificial medium & 18 \\
$70-15-\mathrm{M} 8-1 \sim 18$ & $8^{\text {th }}$ & Artificial medium & 18 \\
$70-15-\mathrm{M} 9-1 \sim 18$ & $9^{\text {th }}$ & Artificial medium & 18 \\
$70-15-\mathrm{M} 10-1 \sim 18$ & $1^{\text {th }}$ & Artificial medium & 18 \\
$70-15-G 0^{\mathrm{c}} 1-1 \sim 10-2$ & - & Artificial medium & 20
\end{tabular}

\begin{tabular}{lcll} 
KJ201 & Parental isolate & 1 \\
KJ201-M1-1 3 & $1^{\text {st }}$ & Artificial medium & 3 \\
KJ201-M2-1 3 & $2^{\text {nd }}$ & Artificial medium & 3 \\
KJ201-M3-1 3 & $3^{\text {rd }}$ & Artificial medium & 3 \\
KJ201-M4-1 3 & $4^{\text {th }}$ & Artificial medium & 3 \\
KJ201-M5-1 3 & $5^{\text {th }}$ & Artificial medium & 3 \\
KJ201-M6-1 3 & $6^{\text {th }}$ & Artificial medium & 3 \\
KJ201-M7-1 3 & $7^{\text {th }}$ & Artificial medium & 3 \\
KJ201-M8-1 3 & $8^{\text {th }}$ & Artificial medium & 3 \\
KJ201-M9-1 3 & $9^{\text {th }}$ & Artificial medium & 3 \\
KJ201-M10-1 3 & $10^{\text {th }}$ & Artificial medium & 3 \\
KJ201-H1-1 3 & $1^{\text {st }}$ & Rice cv. Nakdongbyeo & 3 \\
KJ201-H2-1 3 & $2^{\text {nd }}$ & Rice cv. Nakdongbyeo & 3 \\
KJ201-H3-1 3 & $3^{\text {td }}$ & Rice cv. Nakdongbyeo & 3 \\
KJ201-H4-1 3 & $4^{\text {th }}$ & Rice cv. Nakdongbyeo & 3 \\
KJ201-H5-1 3 & $5^{\text {th }}$ & Rice cv. Nakdongbyeo & 3 \\
KJ201-H6-1 3 & $6^{\text {th }}$ & Rice cv. Nakdongbyeo & 3 \\
KJ201-H7-1 3 & $7^{\text {th }}$ & Rice cv. Nakdongbyeo & 3 \\
KJ201-H8-1 3 & $8^{\text {th }}$ & Rice cv. Nakdongbyeo & 3 \\
KJ201-H9-1 3 & $9^{\text {th }}$ & Rice cv. Nakdongbyeo & 3 \\
KJ201-H10-1 3 & $10^{\text {th }}$ & Rice cv. Nakdongbyeo & 3 \\
\hline Total & & & 238 \\
\hline
\end{tabular}

${ }^{a} 1$ to $10^{\text {th }}$ serial transferred isolates from each parental isolates $70-15$ and KJ201 were recovered by monoconidiation.

${ }^{\mathrm{b}} \mathrm{M}$ means medium-oriented monoconidial isolates; $\mathrm{H}$ means host-oriented monoconidial isolates.

${ }^{c}$ The isolates were generated by cutting the germ tube from the basal and apical cell of a conidium of isolate $70-15$. Therefore, a pair of GT1-1 and GT1-2 was recovered from a conidium.

Amplified fragment length polymorphism (AFLP) analysis. AFLP analysis was performed as described previously (Park et al., 2009) on isolates derived from serial transfers of 70-15 (one isolate from each of 10 generations) and all 20 isolates derived from germ tubes (Table 1). As a first step, $500 \mathrm{ng}$ of genomic DNA was digested with EcoRI and
Table 2. PCR primers used in this study

\begin{tabular}{|c|c|}
\hline Primer & $5^{\prime}-3^{\prime}$ \\
\hline \multicolumn{2}{|c|}{$\begin{array}{l}\text { AVR-Pital_ORF_F ATGCTTTTTTATTCATTATTTTTTTTTCACAC } \\
\text { AVR-Pital_ORF_R TTAACAATATTTATAACGTGCACATTGTG } \\
\text { AVR-Pikm_ORF_F ATGCGTGTTACCACTTTTAACACA } \\
\text { AVR-Pikm_ORF_R TTAAAAGCCGGGCCTTTTTTTCC }\end{array}$} \\
\hline \multicolumn{2}{|c|}{ For AFLP analysis } \\
\hline \multicolumn{2}{|c|}{ Adapter primers } \\
\hline EcoRI-adapter1 & CTCGTAGACTGCGTACC \\
\hline EcoRI-adapter2 & AATTGGTACGCAGTCTAC \\
\hline MspI-adapter1 & GAGTCCTGAGTAGCAG \\
\hline MspI-adapter2 & CGCTCAGGACTCATC \\
\hline \multicolumn{2}{|l|}{ Standard primers } \\
\hline EcoRI-standard & CTCGTAGACTGCGTACCAATTC \\
\hline MspI-standard & GACGATGAGTCCTGAGCGG \\
\hline \multicolumn{2}{|l|}{ Selective primers } \\
\hline EcoRI-0 & GACTGCGTACCAATTC \\
\hline Msp $\mathrm{I}-0$ & GATGAGTCCTGAGCGG \\
\hline EcoRI-ACA & GACTGCGTACCAATTCACA $^{\mathrm{a}}$ \\
\hline MspI-C & GATGAGTCCTGAGCGG들 \\
\hline
\end{tabular}

${ }^{a}$ Underlines are the selective nucleotide.

$M s p I$ and then ligated with $5 \mathrm{pmol}$ of EcoRI and $50 \mathrm{pmol}$ of $M s p I$ double stranded adapters for $2 \mathrm{~h}$ at $16^{\circ} \mathrm{C}$. The ligation mixture was diluted 1:9 and used for preamplification. The selective amplification was performed with four possible combinations of the selective primer pairs (Table 2). The amplified samples were loaded in a polyacrylamide gel and stained with Vistra Green (Amersham Pharmacia Biotech, Piscataway, NJ, USA) as described previously (Park et al., 2009). Fragment polymorphics compared to parental haplotype were excised under a UV transilluminator and were recovered by reamplification as described previously (Park et al., 2009) and sequenced. The identity of recovered fragments was compared to sequence databases using the BLAST Network Service (National Center for Biotechnology Service).

\section{Results and Discussion}

To investigate the genetic stability of $M$. oryzae during serial transfers, we systematically collected conidia during 10 serial passages of two M. oryzae isolates both in vitro and in vivo. One hundred fifty six single-conidial isolates were generated from isolate 70-15 and 30 isolates from KJ201 by successively transferring these isolates on OMA for 10 generations (Fig. 1A). An additional 30 isolates were collected from serial inoculations of rice cv. Nakdongbyeo with isolate KJ201 (Fig. 1B). Additionally, 20 isolates were generated in vitro from germ tubes originating from both the basal and apical cells from 10 conidia of isolate $70-15$ (Fig. 1D). A total of 238 isolates, including parental isolates 

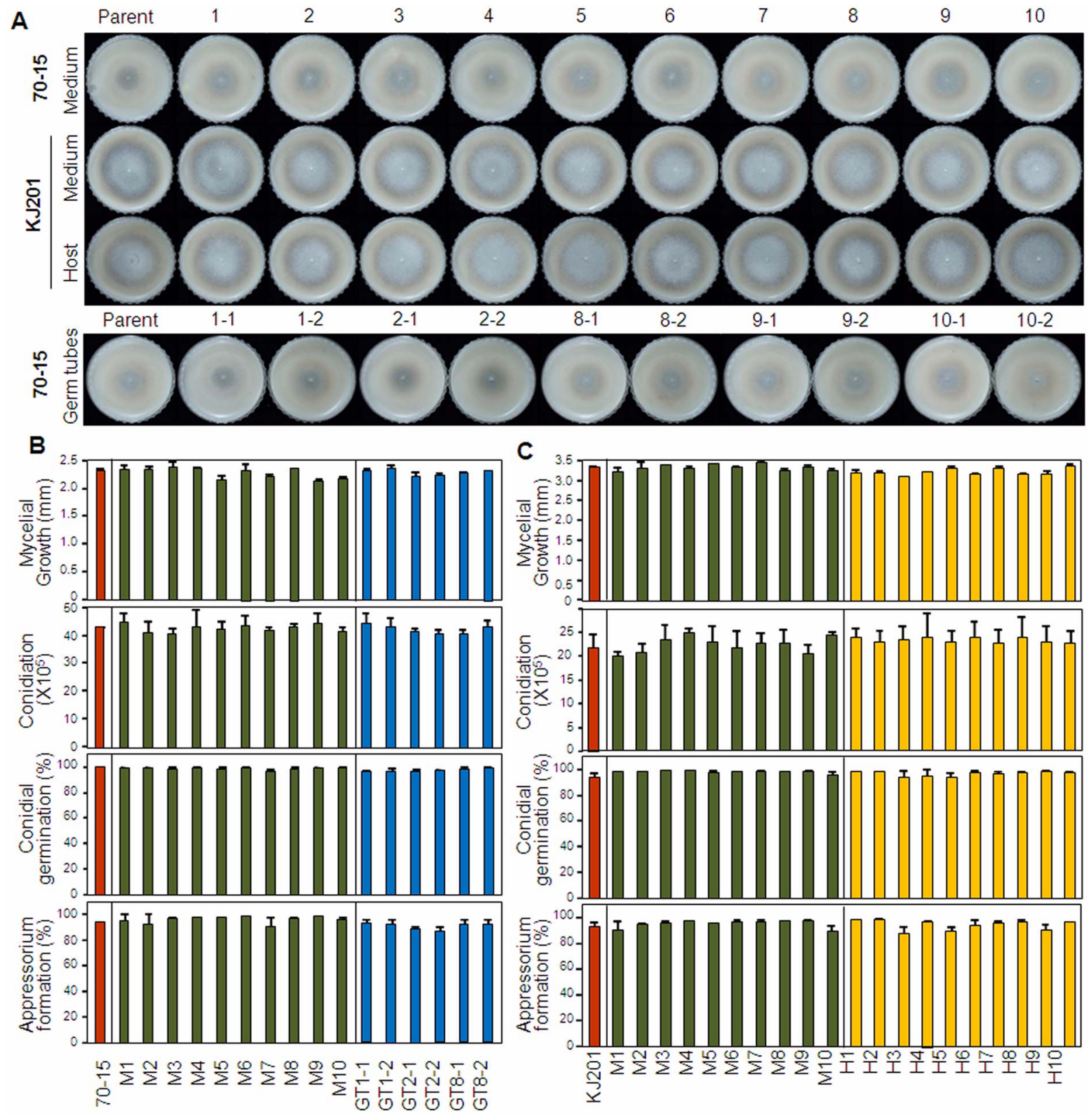

Fig. 2. Phenotypic characterization of M. oryzae after serial passage on medium and through host plants. (A) Cultural characteristics of parental isolates 70-15 and KJ201 and isolates derived from them after serial passages. Cultures were photographed 7 days after inoculation on oatmeal agar medium (OMA). Numbers 1 to 10 indicate $1^{\text {st }}$ to $10^{\text {th }}$ serial transfer on medium and rice plants. GT indicates isolates that were recovered from the germ tubes at the basal and apical cells of 10 conidia; 1(to 10)-1 and 1(to 10)-2 were generated from apical and basal cells, respectively. (B) Mycelial growth, conidiation, conidial germination, and appressorium formation of isolate 70-15, single-conidial isolates derived from it after successive serial transfers on OMA (M), and representatives of three pairs of isolates from germinated conidia (GT). (C) Mycelial growth, conidiation, conidial germination, and appressorium formation of isolate KJ201, singleconidial isolates derived from it after successive serial transfers on OMA $(M)$ and through rice plants $(H)$.

70-15 and KJ201, were subjected to phenotypic and genotypic analyses (Table 1).

We observed no significant differences between the parental isolates and any isolates derived from them in successive generations through both medium and host plants for any of the six phenotypes tested in vitro. No differences were observed for mycelial growth or cultural morphology on three different media, potato dextrose agar, CM and OMA (Fig. 2A, 2B, and 2C), for conidiation on V8 juice agar and OMA media, or for conidial morphology, conidial germination, and appressorium formation. In addition, we did not found any sectoring either from medium 
transfers or host transfers.

Similarly, no differences were observed for virulence in planta. All tested isolates were highly virulent to $\mathrm{cv}$. Nakdongbyeo, which is highly susceptible to KJ201 and 70-15 (Chi et al., 2009; Jeon et al., 2007), with disease indexes of 7-9 (maximum of 9), indicating that virulence was not significantly affected (Fig. 3A). These results are similar to those of Latterell and Rossi (1986), who reported no loss of virulence and no changes in cultural characteristics in M. oryzae isolates after repeated assays of the same isolates from stock cultures over long periods of time. However, because stability of virulence does not guarantee stability of pathotype in M. oryzae, pathogenicity assays of differential rice lines that contain different blast resistance genes are needed to detect variation in pathotypes.

To assess genotypic stability during serial transfers, we conducted DNA fingerprinting with various probes including transposable elements (MGR586, Pot2, MAGGY, LINE, and Mg-SINE), a host specificity gene (PWL2), and an avirulence gene (AVR-Pital). Isolates 70-15 and KJ201 differed in DNA fingerprint haplotypes when MGR586 and MAGGY were used as probes, but all isolates derived from them were identical to their parent isolates. Representative autoradiograms of blots probed with MGR586 and MAGGY for isolate $\mathrm{KJ} 201$ and its single-conidial isolates are shown in Figs. 3B and 3C, respectively. The other probes, including Mg-SINE (Kachroo et al., 1995), LINE (Nishimura et al., 2000), Pot2 (Kachroo et al., 1994), host specificity gene PWL2 (Sweigard et al., 1995), and avirulence gene AVRPital (Khang et al., 2008), also failed to detect differences among isolates (Fig. 3E, F, and G). These results indicate that these transposable elements were stably maintained during 10 serial passages.

Interestingly, however, an approximately 8 -kb restriction fragment that is present in parental isolate KJ201 was absent in several single-conidial isolates when the telomerehelicase gene TLH1 (Gao et al., 2002) was used as a probe (Fig. 3D). One reversible loss of a TLH1 fragments was observed during successive transfers (Fig. 3D).

We also determined the stability of two telomere-located
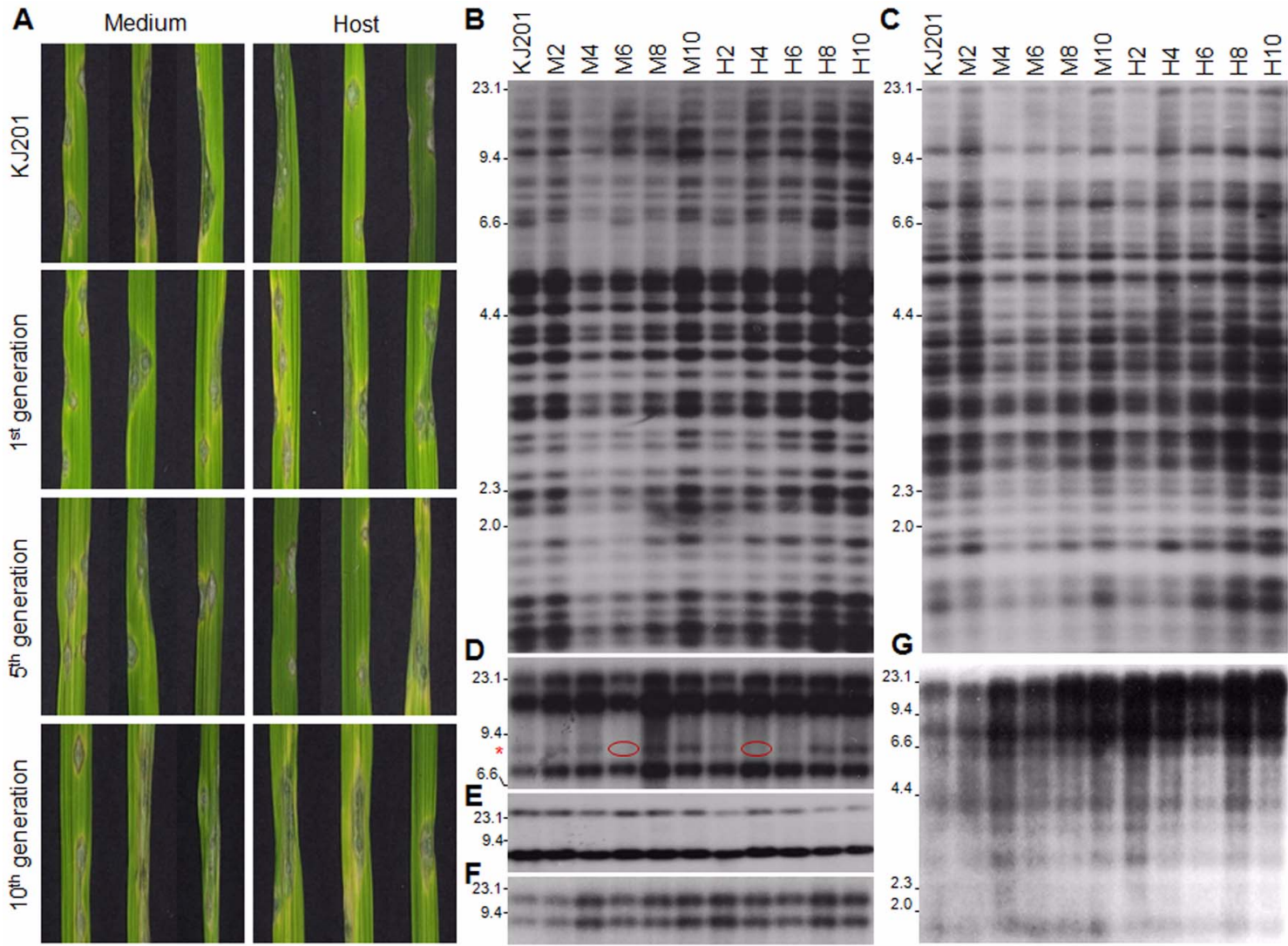

Fig. 3. Virulence assay and DNA fingerprinting. (A) Virulence assay using isolates from the $1^{\text {st }}, 5^{\text {th }}$, and $10^{\text {th }}$ serial transfers of isolate KJ201. Rice seedlings (cv. Nakdongbyeo) were inoculated at the 3 4-leaf stage, with three replicates. (B) DNA fingerprinting of isolates from $2^{\text {nd }}, 4^{\text {th }}, 6^{\text {th }}, 8^{\text {th }}$, and $10^{\text {th }}$ serial transfers of isolate KJ201 on medium $(\mathrm{M})$ and host plants $(\mathrm{H})$. The number after $\mathrm{M}$ and $\mathrm{H}$ indicates generation number of serial transfers for each isolate. Probes included (B) MGR586, (C) MAGGY, (D) Telomere-linked helicase 1 (TLH1), (E) AVR-Pital, (F) PWL2, and (G) LINE. The asterisk in (D) denotes a restriction fragment for a member of the $T L H$ gene family that was lost in two isolates (M6 and H4). 
avirulence genes including AVR-Pital (Chomosome 3) (Orbach et al., 2000) and AVR-Pikm (Chomosome 1) (Feng et al., 2007) at the nucleotide sequence level. The coding regions of the AVR-Pital and AVR-Pikm were sequenced in parental isolate $\mathrm{KJ} 201$ and single-conidial isolates after the $1^{\text {st }}, 5^{\text {th }}$ and $10^{\text {th }}$ serial passages on medium and on plant hosts. However, no sequence variation was detected.

We further analyzed a sample of isolates using AFLPs to detect any additional genetic variation. Twenty isolates from germ tubes of 10 conidia and one representative isolate from each generation of serial transfers of isolate 70-15 were genotyped. Four AFLP primer pairs were used. Polymorphism was detected in only one isolate, 70-15-GT 8-1, generated from the basal cell of a conidium. Sequence analysis of this polymorphic band showed that it matched with partial fragment of a solo LTR (37-bp, AATTCTTCGTGACGGAAACCAAGTTCCTGGGCCTGCT) that is highly dispersed in the M. oryzae genome. This suggests that some transposable elements such as solo LTR are mobile and might be agents of genetic variation in M. oryzae. However, we have not found any additional phenotypic and genotypic changes in isolate 70-15-GT 8-1.

In conclusion, we conducted 10 serial transfers of two rice-pathogenic isolates of $M$. oryzae, 70-15 and KJ201. All single-conidial isolates showed stable genotypes, with minor exceptions, and stable phenotypes, including virulence.

\section{Acknowledgments}

This work was supported by grants from the National Research Foundation of Korea (2009-0063340 and 20090080161), the Biogreen21 (20080401-034-044-009-01-00), the TDPAF (309015-04-SB020), and the Crop Functional Genomics Center (2009K001198).

\section{References}

Bonman, J. M., Vergel de Dois, T. I. and Khin, M. M. 1986. Physiologic specialization of Pyricularia oryzae in the Philippines. Plant Dis. 70:767-769.

Borromeo, E. S., Nelson, R. J., Bonman, J. M. and Leung, H. 1993. Genetic differentiation among isolates of Pyricularia infecting rice and weed hosts. Phytopathology 83:393-399.

Chen, D., Zeigler, R. S., Leung, H. and Nelson, R. J. 1995. Population structure of Pyricularia grisea at two screening sites in the Philippines. Phytopathology 85:1011-1020.

Chi, M. H., Park, S. Y., Kim, S. and Lee, Y. H. 2009. A novel pathogenicity gene is required in the rice blast fungus to suppress the basal defenses of the host. PLoS Pathog. 5: e1000401.

Correa-Victoria, F. J. and Zeigler, R. S. 1993. Field breeding for durable rice blast resistance in the presence of diverse pathogen populations. Edited by Jacobs, T \& Parleviet, JR, Durabil- ity of Disease Resistance. Norwell: Kluwer Academic Publishers.

Correa-Victoria, F. K. and Zeigler, R. S. 1993. Pathogenic variability in Pyricularia grisea at a rice blast "hot spot" breeding site in eastern Colombia. Plant Dis. 77:1029-1035.

Dean, R. A., Talbot, N. J., Ebbole, D. J. Farman, M. L., Mitchell, T. K., Orbach, M. J., Thon, M., Kulkarni, R., Xu, J. R., Pan, H., Read, N. D., Lee, Y. H., Carbone, I., Brown, D., Oh, Y. Y., Donofrio, N., Jeong, J. S., Soanes, D. M., Djonovic, S., Kolomiets, E., Rehmeyer, C., Li, W., Harding, M., Kim, S., Lebrun, M. H., Bohnert, H., Coughlan, S., Butler, J., Calvo, S., Ma, L. J., Nicol, R., Purcell, S., Nusbaum, C., Galagan, J. E., and Birren, B. W. 2005. The genome sequence of the rice blast fungus Magnaporthe grisea. Nature 434:980-986.

Feng, S., Ma, J., Lin, F., Wang, L. and Pan, Q. 2007. Construction of an electronic physical map of Magnaporthe oryzae using genomic position-ready SSR markers. Chin. Sci. Bull. 52: 3346-3354.

Gao, W., Khang, C. H., Park, S.-Y., Lee, Y.-H. and Kang, S. 2002. Evolution and organization of a highly dynamic, subtelomeric helicase gene family in the rice blast fungus Magnaporthe grisea. Genetics 162:103-112.

Genovesi, A. D. and Magill, C. W. 1976. Heterokaryosis and parasexuality in Pyricularia oryzae Cavara. Can. J. Microbiol. 22:531-536.

Han, S. S., Ra, D. S., Choi, S. H. and Kim, C. K. 1997. Population dynamics of Pyricularia grisea during leaf and panicle blast stages in the same field. Korean J. Plant Pathol. 13:408-415.

Jeon, J., Goh, J., Yoo, S., Chi, M. H., Choi, J., Rho, H. S., Park, J., Han, S. S., Kim, B. R., Park, S. Y., Kim, S., and Lee, Y. H. 2008. A putative MAP kinase kinase kinase, $M C K 1$, is required for cell wall integrity and pathogenicity of the rice blast fungus, Magnaporthe oryzae. Mol. Plant-Microbe. Interact. 21:525-534.

Jeon, J., Park, S. Y., Chi, M. H., Choi, J., Park, J., Rho, H. S., Kim, S., Goh, J., Yoo, S., Park, J. Y., Yi, M., Yang, S., Kwon, M. J., Han, S. S., Kim, B. R., Khang, C. H., Park, B., Lim, S. E., Jung, K., Kong, S., Karunakaran, M., Oh, H. S., Kim, H., Kang, S., Choi, W. B., and Lee, Y. H. 2007. Genome-wide functional analysis of pathogenicity genes in the rice blast fungus. Nat. Genet. 39:561-565.

Kachroo, P., Leong, S. A. and Chattoo, B. B. 1994. Pot2, an inverted repeat transposon from the rice blast fungus Magnaporthe grisea. Mol. Gen. Genet. 245:339-348.

Kachroo, P., Leong, S. A. and Chattoo, B. B. 1995. Mg-SINE: a short interspersed nuclear element from the rice blast fungus, Magnaporthe grisea. Proc. Natl. Acad. Sci. USA 92:1112511129.

Kang, S. and Lee, Y.-H. 2000. Population structure and race variation of the rice blast fungus. Plant Pathol. J. 16:1-8.

Kang, S., Lebrun, M.-H., Farrall, L. and Valent, B. 2001. Gain of virulence caused by insertion of a Pot3 transposon in a Magnaporthe grisea avirulence gene. Mol. Plant- Microbe Interact. 14:671-674.

Khang, C. H., Park, S. Y., Lee, Y. H., Valent, B. and Kang, S. 2008. Genome organization and evolution of the AVR-Pita 
avirulence gene family in the Magnaporthe grisea species complex. Mol. Plant-Microbe Interact. 21:658-670.

Latterell, F. M. and Rossi, A. E. 1986. Longevity and pathogenic stability of Pyricularia oryzae. Phytopathology 76:231-235.

Levy, M., Correa-Victoria, F. J., Zeigler, R. S., Hu, S. and Hamer, J. E. 1993. Genetic diversity of the rice blast fungus in a disease nursery in Colombia. Phytopathology 83:1427-1433.

Nishimura, M., Hayashi, N., Jwa, N. S., Lau, G. W., Hamer, J. E. and Hasebe, A. 2000. Insertion of the LINE retrotransposon MGL causes a conidiophore pattern mutation in Magnaporthe grisea. Mol. Plant Microbe-Interact. 13:892-894.

Noguchi, M. T., Yasuda, N. and Fujita, Y. 2006. Evidence of genetic exchange by parasexual recombination and genetic analysis of pathogenicity and mating type of parasexual recombinants in rice blast fungus. Magnaporthe oryzae. Phytopathology 96:746-750.

Orbach, M. J., Farrall, L., Sweigard, J. A., Chumley, F. G. and Valent, B. 2000. A telomeric avirulence gene determines efficacy for rice blast resistance gene $\mathrm{Pi-ta}$. Plant Cell 12:2019-2032.

Park, S.-Y., Jwa, N. S., Chi, M.-H. and Lee, Y.-H. 2009. A fluorescence-based cDNA-AFLP method for identification of differentially expressed genes. Plant Pathol. J. 25:184-188.

Park, S.-Y., Milgroom, M. G., Han, S. S., Kang, S. and Lee, Y.-H. 2003. Diversity of pathotypes and DNA fingerprint haplotypes in populations of Magnaporthe grisea in Korea over two decades. Phytopathology 93:1378-1385.

Rao, K. M. 1994. Rice blast disease. Delhi-119935: Daya publishing house.
Sweigard, J. A., Carroll, A. M., Kang, S., Farrall, L., Chumley, F. G. and Valent, B. 1995. Identification, cloning, and characterization of $P W L 2$, a gene for host species specificity in the rice blast fungus. Plant Cell 7:1221-1233.

Valent, B., Farrall, L., and Chumley, F. G. 1991. Magnaporthe grisea genes for pathogenicity and virulence identified through a series of backcrosses. Genetics 127:87-101.

Wu, B. C. and Magill, C. W. 1995. Spontaneous mutations at fingerprint loci in clonal lineages of the rice blast fungus. Exp. Mycol. 19:86-90.

Xia, J. Q. and Correll, J. C. 1995. Examination of mitotic stability and hybridization potential between two genetically distinct haplotypes of Magnaporthe grisea. Exp. Mycol. 19:171-177.

Xia, J. Q., Correll, J. C., Lee, F. N. and Ross, W. J. 2000. Regional population diversity of Pyricularia grisea in Arkansas and the influence of host selection. Plant Dis. 84:877-884.

Xia, J. Q., Correll, J. C., Lee, F. N., Marchetti, M. A. and Rhoads, D. D. 1993. DNA fingerprinting to examine microgeographic variation in the Magnaporthe grisea (Pyricularia grisea) population in two rice fields in Arkansas. Phytopathology 83: 1029-1035.

Zeigler, R. S., Scott, R. P., Leung, H., Bordeos, A. A., Kumar, J. and Nelson, R. J. 1997. Evidence of parasexual exchange of DNA in the rice blast fungus challenges its exclusive clonality. Phytopathology 87:284-294.

Zhou, E., Jia, Y., Singh, P., Correll, J. and Lee, F. N. 2007. Instability of the Magnaporthe oryzae avirulence gene AVR-Pita alters virulence. Fungal Genet. Biol. 44:1024-1034. 